Article

\title{
Influence of Hydrothermal Treatment on Physicochemical Properties and Drug Release of Anti-Inflammatory Drugs of Intercalated Layered Double Hydroxide Nanoparticles
}

\author{
Zi Gu, Aihua Wu, Li Li and Zhi Ping Xu *
}

Australian Institute of Bioengineering and Nanotechnology, the University of Queensland, Brisbane, QLD 4072, Australia; E-Mails: z.gu@uq.edu.au (Z.G.); aihua.wu@uq.net.au (A.W.); 1.1i2@uq.edu.au (L.L.)

* Author to whom correspondence should be addressed; E-Mail: gordonxu@uq.edu.au; Tel.: +61-7-334-63809; Fax: +61-7-334-63973.

Received: 3 April 2014; in revised form: 8 May 2014 / Accepted: 9 May 2014 /

Published: 22 May 2014

\begin{abstract}
The synthesis method of layered double hydroxides (LDHs) determines nanoparticles' performance in biomedical applications. In this study, hydrothermal treatment as an important synthesis technique has been examined for its influence on the physicochemical properties and the drug release rate from drug-containing LDHs. We synthesised MgAl-LDHs intercalated with non-steroidal anti-inflammatory drugs (i.e., naproxen, diclofenac and ibuprofen) using a co-precipitation method with or without hydrothermal treatment $\left(150{ }^{\circ} \mathrm{C}, 4 \mathrm{~h}\right)$. After being hydrothermally treated, LDH-drug crystallites increased in particle size and crystallinity, but did not change in the interlayer anion orientation, gallery height and chemical composition. The drug release patterns of all studied LDH-drug hybrids were biphasic and sustained. LDHs loaded with diclofenac had a quicker drug release rate compared with those with naproxen and ibuprofen, and the drug release from the hydrothermally-treated LDH-drug was slower than the freshly precipitated LDH-drug. These results suggest that the drug release of LDH-drugs is influenced by the crystallite size of LDHs, which can be controlled by hydrothermal treatment, as well as by the drug molecular physicochemical properties.
\end{abstract}

Keywords: layered double hydroxides; hydrothermal treatment; nonsteroidal anti-inflammatory drugs; physicochemical properties; drug release 


\section{Introduction}

Layered double hydroxides (LDHs), also known as hydrotalcite-like materials or anionic clays, can be found in nature as minerals, as well as being readily synthesised in the laboratory [1]. The general chemical formula of LDHs is $\left[\mathrm{M}^{2+}{ }_{1-x} \mathrm{M}^{3+}{ }_{x}(\mathrm{OH})_{2}\right]^{x+}\left(\mathrm{A}^{n-}\right)_{x / n} \cdot m \mathrm{H}_{2} \mathrm{O}$, where $x=0.2-0.33$, indicating that the $\mathrm{Mg} / \mathrm{Al}$ molar ratio is $c a .2 .0-4.0$, and $m=1-3 x / 2$ [1]. They consist of positively charged brucite-like layers $\left(\left[\mathrm{M}^{2+}{ }_{1-x} \mathrm{M}^{3+}{ }_{x}(\mathrm{OH})_{2}\right]^{x+}\right)$ and the interlayer neutralising anions $\left(\left(\mathrm{A}^{n-}\right)_{x / n}\right)$, as well as $\mathrm{H}$-bonded water molecules $\left(m \mathrm{H}_{2} \mathrm{O}\right)$.

$\mathrm{LDH}$ is one of the important emerging nano-carriers for therapeutic molecule delivery, due to their merits, such as the ease and low-cost of preparation, good biocompatibility, low cytotoxicity, protection for the intercalated drugs and the capacity to facilitate the loaded drugs in escaping from the endosome [2-4]. A number of drugs/genes have been loaded on/into LDHs and have shown enhanced biological or therapeutic effects on the prevention and treatment of cancer [5-7], cardiovascular disease [8-11] and inflammation [12-15].

LDH nanoparticles can be synthesised by various methods [1]. The most commonly used method is the co-precipitation of mixed metal salt solution in hydroxide solution at a constant or various $\mathrm{pH}$ followed by aging at a temperature over $100{ }^{\circ} \mathrm{C}$ (hydrothermal treatment) [1]. Xu et al. described the hydrothermal treatment as a process involving several events, such as disaggregation, particle growth, and re-aggregation, which occur in series and/or in parallel [16]. The high temperature increases the Brownian motion of LDH particles and enables the particles escape from the aggregate to be suspended as individual nanoparticles. Hydrothermal treatment also makes the metal cations $\left(\mathrm{M}^{2+}\right.$ and $\left.\mathrm{M}^{3+}\right)$ distribute more evenly within the hydroxide layers and form a better crystallised LDH particle. Meanwhile, as the particles grow in the hydrothermal environment, prolonged hydrothermal treatment may cause re-aggregation, due to the formation of big particles overcoming the electrostatic repulsion between particles. Labajos et al. found that hydrothermal treatment led to an increase in the $\mathrm{Mg} / \mathrm{Al}$ ratio, a decrease in the water content and a more ordered structure of the interlayer species [17].

Although there are a few studies on the influences of hydrothermal treatment on the physicochemical properties of LDHs, little is known on the changes in the physicochemical and release properties of therapeutic molecule-intercalated LDH nanohybrids after being hydrothermally treated. In this study, we synthesised MgAl-LDH-containing non-steroidal anti-inflammatory drugs (i.e., naproxen, diclofenac or ibuprofen) with or without hydrothermal treatment. The aim is to investigate the effect of hydrothermal treatment on the morphology, particle size and structure of the drug-LDH nanohybrids, as well as the subsequent possible changes in the drug release pattern.

\section{Materials and Methods}

\subsection{Materials}

Phosphate buffered saline (PBS, pH 7.4, consisting of $1 \mathrm{mM} \mathrm{KH}_{2} \mathrm{PO}_{4}, 155 \mathrm{mM} \mathrm{NaCl}$ and $3 \mathrm{mM}$ $\mathrm{Na}_{2} \mathrm{HPO}_{4} \cdot 7 \mathrm{H}_{2} \mathrm{O}$ ) was purchased from Life Technologies (Grand Island, NY, USA) and other chemicals from Sigma-Aldrich (St. Louis, MO, USA) with a purity of $97 \%-99 \%$. Milli-Q water was used in all experiments. 


\subsection{Synthesis of Drug-Containing Layered Double Hydroxides (LDHs)}

Drug-containing LDHs were prepared using a co-precipitation method with or without hydrothermal treatment. Prior to mixing the solutions, all solutions were heated to $80{ }^{\circ} \mathrm{C}$ with nitrogen gas bubbling for $20 \mathrm{~min}$. A solution of $\mathrm{NaOH}(40 \mathrm{~mL}, 0.15 \mathrm{M})$ and a mixed solution $(10 \mathrm{~mL})$ containing $\mathrm{Mg}\left(\mathrm{NO}_{3}\right)_{2} \cdot 6 \mathrm{H}_{2} \mathrm{O}(0.2 \mathrm{M})$ and $\mathrm{Al}\left(\mathrm{NO}_{3}\right)_{3} \cdot 9 \mathrm{H}_{2} \mathrm{O}(0.1 \mathrm{M})$ were added dropwise to a drug solution $(10 \mathrm{~mL}$, $0.1 \mathrm{M}$ ) containing naproxen (NAP) sodium, diclofenac (DIC) sodium or ibuprofen (IBU) sodium at $80{ }^{\circ} \mathrm{C}$ with nitrogen gas purging. Throughout the addition, the $\mathrm{pH}$ value of the mixture suspension was maintained at $11.0 \pm 0.5$. The mixture was continuously stirred for $10 \mathrm{~min}$ at $80{ }^{\circ} \mathrm{C}$ under $\mathrm{N}_{2}$ gas purging. The resulting mixture was separated, washed and dispersed in $40 \mathrm{~mL}$ deionised water. The resultant drug-containing LDH suspension was divided into two parts. One part of the suspension was hydrothermally treated at $150{ }^{\circ} \mathrm{C}$ for $4 \mathrm{~h}$ and named as LDH-drug-HT (i.e., LDH-NAP-HT, LDH-DIC-HT and LDH-IBU-HT, respectively). The other part was freshly precipitated without hydrothermal treatment, and named as LDH-drug-FP (i.e., LDH-NAP-FP, LDH-DIC-FP and LDH-IBU-FP, respectively). As-obtained drug-LDH hybrids were then collected via high-speed centrifugation and dried in a $50{ }^{\circ} \mathrm{C}$ oven for a few days for further characterisations.

\subsection{Drug Release from Drug-Containing $L D H S$}

LDH-drug hybrid powder (160 mg) was suspended in $80 \mathrm{~mL} \mathrm{pH} 7.4$ PBS in a sealed flask and shaken at $170 \mathrm{rpm}$ in a water bath at $37^{\circ} \mathrm{C}$. An aliquot of the medium solution $(0.5 \mathrm{~mL})$ was withdrawn at certain time points and replaced with $0.5 \mathrm{~mL}$ of fresh PBS. The aliquot was centrifuged twice to remove possible nanoparticles. After 5 days of release, the concentration of released naproxen, diclofenac or ibuprofen in the aliquot sample was determined by measuring absorbance at $\lambda=320,300$ or $264 \mathrm{~nm}$.

\subsection{Characterisations}

The average hydrodynamic particle size and the size distribution were measured by dynamic light scattering (DLS) on a Nanosizer Nano Zetasizer instrument (Malvern Instruments, Malvern, UK), after the particles were dispersed in ethanol via ultrasonication for $30 \mathrm{~min}$. The lateral diameter of the LDH nanoparticle was examined by scanning electron microscopy (SEM) on a JEOL 6300 SEM. LDH samples were dispersed in $75 \%$ ethanol solution via ultrasonication for $10 \mathrm{mins}$, and then, the SEM images were taken at 10-15 kV with magnifications of 20,000-80,000. Powder X-ray diffraction (XRD) patterns were recorded on a Rigaku Miniflex X-ray Diffractometer using Co $K \alpha$ source $(\lambda=0.178897 \mathrm{~nm})$ at a scanning rate of $0.02^{\circ} / \mathrm{s}(2 \theta)$ from $2 \theta=2^{\circ}$ to $2 \theta=80^{\circ}$. Fourier transform infrared (FTIR) spectra were obtained on a Nicolet 6700 FTIR (Thermo Scientific, Waltham, MA, USA) in the range of $4000-400 \mathrm{~cm}^{-1}$ by accumulating 32 scans at a resolution of $4 \mathrm{~cm}^{-1}$. Inductively coupled plasma optical emission spectrometry (ICP-OES) was conducted on a Varian axial Vista CCD Simultaneous (Varian, Mulgrave, Australia) with the wavelength used for Mg and Al at 383.829 and $237.312 \mathrm{~nm}$, respectively. The content of C, N and H was measured on a CHNS-O analyser (Flash EA 1112 Series, Thermo Scientific). The drug loading capacity was calculated as the drug mass divided by the LDH-drug complex mass. 


\subsection{Computational Simulation}

All geometries were optimised based on the density functional theory [18], within the generalised gradient approximation, together with the widely employed Perdew-Burke-Ernzerhof function [19], as embedded in the DMol3 code [20,21]. During the calculations, all structures were fully relaxed until the total energy was converged to $2.0 \times 10^{-5} \mathrm{eV} /$ atom. The drug molecular anion orientation between two LDH brucite-like layers was determined based on the following assumption: (1) the molecule is adsorbed on the LDH brucite-like layers through the equal bonding of the two oxygen atoms in the $\mathrm{COO}^{-}$group; and (2) the $\mathrm{COO}^{-}$plane is vertically bonded onto the $\mathrm{LDH}$ layer, and the left part is fully relaxed, where the axis of the benzene rings form a dihedral angle, $\theta$, that defines the orientation of the drug molecule in the LDH interlayer (e.g., $\theta=0^{\circ}$ and $90^{\circ}$ indicating that the molecule is parallel and perpendicular to the surface, respectively).

\section{Results and Discussion}

\subsection{LDH-Drug Particle Size}

The LDH-drug hybrids were white, turbid dispersion with or without hydrothermal treatment. Ultrasonic treatment was used to break down the dried agglomerates of LDH-NAP, LDH-DIC and LDH-IBU in ethanol for the particle size distribution measurement, and all LDH-drug suspension had a narrow size distribution with a polydispersity index (PDI) smaller than 0.3 (Table 1). Obviously, in the case of LDH containing NAP or DIC, the average hydrodynamic diameter of LDH-drug particles prepared with hydrothermal treatment (194-332 nm) was significantly larger than that without hydrothermal treatment (159-172 nm) (Table 1).

Table 1. Average particle size and polydispersity index (PDI) of layered double hydroxide (LDH) drug measured by dynamic light scattering (DLS) and average lateral diameter by scattering electron microscopy (SEM). Data are expressed as the mean \pm SD (nm). A $t$-test was performed between LDH-drug-HT (hydrothermally treated) and LDH-drug-FP (freshly precipitated), and $p<0.05$ was considered statistically significant $(*)$. NAP, naproxen; DIC, diclofenac; IBU, ibuprofen.

\begin{tabular}{cccc}
\hline Sample & Source of data & HT $(\mathbf{n m})$ & FP $(\mathbf{n m})$ \\
\hline \multirow{2}{*}{ LDH-NAP } & DLS & $332 \pm 36 / 0.19 \pm 0.04$ & $172 \pm 12 / 0.26 \pm 0.02$ \\
& SEM & $230 \pm 60$ & $58 \pm 22^{*}$ \\
\hline \multirow{2}{*}{ LDH-DIC } & DLS & $194 \pm 11 / 0.28 \pm 0.02$ & $159 \pm 18 / 0.20 \pm 0.02$ \\
& SEM & $168 \pm 34$ & $54 \pm 13^{*}$ \\
\hline \multirow{2}{*}{ LDH-IBU } & DLS & $342 \pm 14 / 0.09 \pm 0.00$ & $272 \pm 44 / 0.25 \pm 0.06$ \\
& SEM & $270 \pm 71$ & $88 \pm 19^{*}$ \\
\hline
\end{tabular}

SEM images in Figure 1 demonstrated the morphology and particle lateral diameter of the LDH-drug samples. All LDH nanoparticles had a disc-like shape with a relatively homogenous distribution. By measuring the lateral diameter of 10 particles that were randomly selected in two SEM images for each sample, LDH-drug-HT had a significantly larger average diameter than LDH-drug-FP (Table 1), which was consistent with the observation by dynamic light scattering (DLS). 
Figure 1. TEM images of LDH-NAP-HT (A); LDH-NAP-FP (B); LDH-DIC-HT (C); LDH-DIC-FP (D); LDH-IBU-HT (E); and LDH-IBU-FP (F). Scale bar: $500 \mathrm{~nm}$.
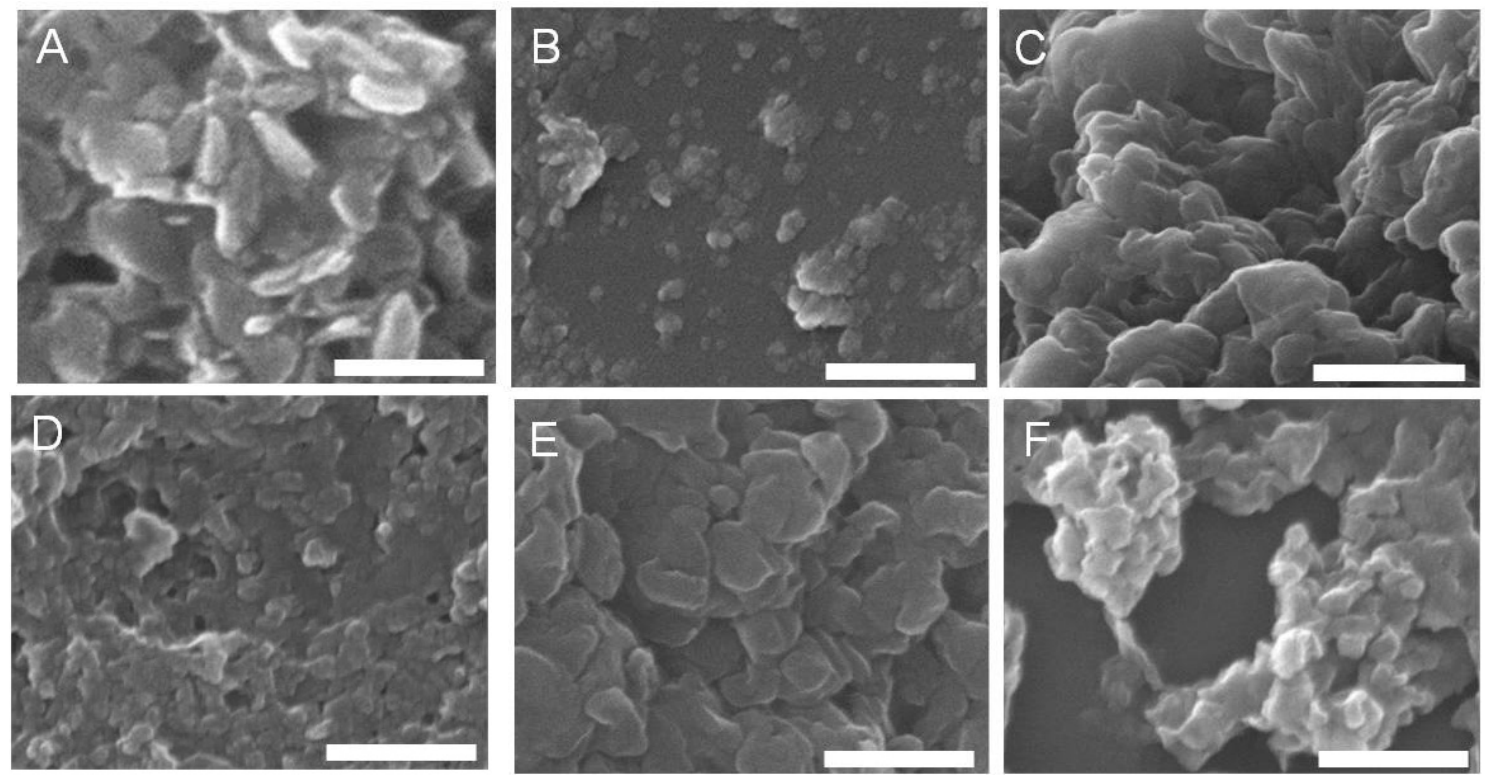

The hydrodynamic diameter of LDH-NAP-HT, LDH-NAP-FP, LDH-DIC-FP and LDH-IBU-FP measured by DLS was significantly larger than the corresponding lateral diameter by SEM (Table 1). However, for LDH-DIC-HT and LDH-IBU-HT, whereas reported by $\mathrm{Xu}$ et al. [22], the lateral dimension of LDHs in SEM images was similar to the average particle size from DLS. The larger particle size from DLS thus suggests that aggregates were not completely dispersed into individual crystals in the DLS samples despite the ultrasonic treatment applied. In particular, the LDH-drug samples prepared without hydrothermal treatment were severely agglomerated, with the apparent average size of agglomerated particles being 3-4 times larger (e.g., 20-50 crystallites in one aggregate). In comparison, the average particle size of LDH-drug-HT from SEM was just slightly smaller than that from DLS (Table 1), suggesting that hydrothermal treatment facilitated LDH-drug particles to disperse. We believe that the average particle size from SEM reflects the true crystal size of LDH-drug nanoparticles. Based on the SEM observation, hydrothermal treatment thus generated approximately three-times larger particles for LDH-NAP, LDH-DIC and LDH-IBU (Table 1). For example, LDH-DIC with or without hydrothermal treatment $\left(150^{\circ} \mathrm{C}, 4 \mathrm{~h}\right)$ had a lateral diameter of $168 \pm 34$ and $54 \pm 13 \mathrm{~nm}$, respectively.

\subsection{LDH-Drug Structure and Composition}

The powder X-ray diffraction (XRD) patterns (Figure 2) showed that all LDH-drug samples were typical lamellar materials [9], characterised with a series of basal diffractions at low $2 \theta$ angles and weaker non-basal diffractions at higher angles. LDH-drug-HT had obviously smaller full width at half-maximum (FWHM) $\left(0.46^{\circ}-0.5^{\circ}\right)$ than $\mathrm{LDH}-\mathrm{drug}-\mathrm{FP}\left(0.76^{\circ}-0.88^{\circ}\right)$ (Table 2$)$. The narrow peaks after hydrothermal treatment, e.g., the smaller FWHM values, indicated the increased thickness of LDH sheet-like crystals (along the $c$-axis) [23]. This was in agreement with the average lateral particle size from SEM images (Figure 1 and Table 1) if the lateral-to-height aspect ratio was supposedly similar before and after hydrothermal treatment. In addition, hydrothermal treatment generated much 
better defined basal diffraction peaks, suggesting improved crystallinity [24]. The improved crystallinity of LDH-IBU after hydrothermal treatment was also reported previously by Gunawan and Xu, who showed that hydrothermal treatment at $150{ }^{\circ} \mathrm{C}$ for $18 \mathrm{~h}$ exhibited a higher degree of crystallinity than aging under at $70{ }^{\circ} \mathrm{C}$ for three days [25].

Figure 2. Powder X-ray diffraction (XRD) patterns for LDH-NAP-HT/FP, LDH-DIC-HT/FP and LDH-IBU-HT/FP.

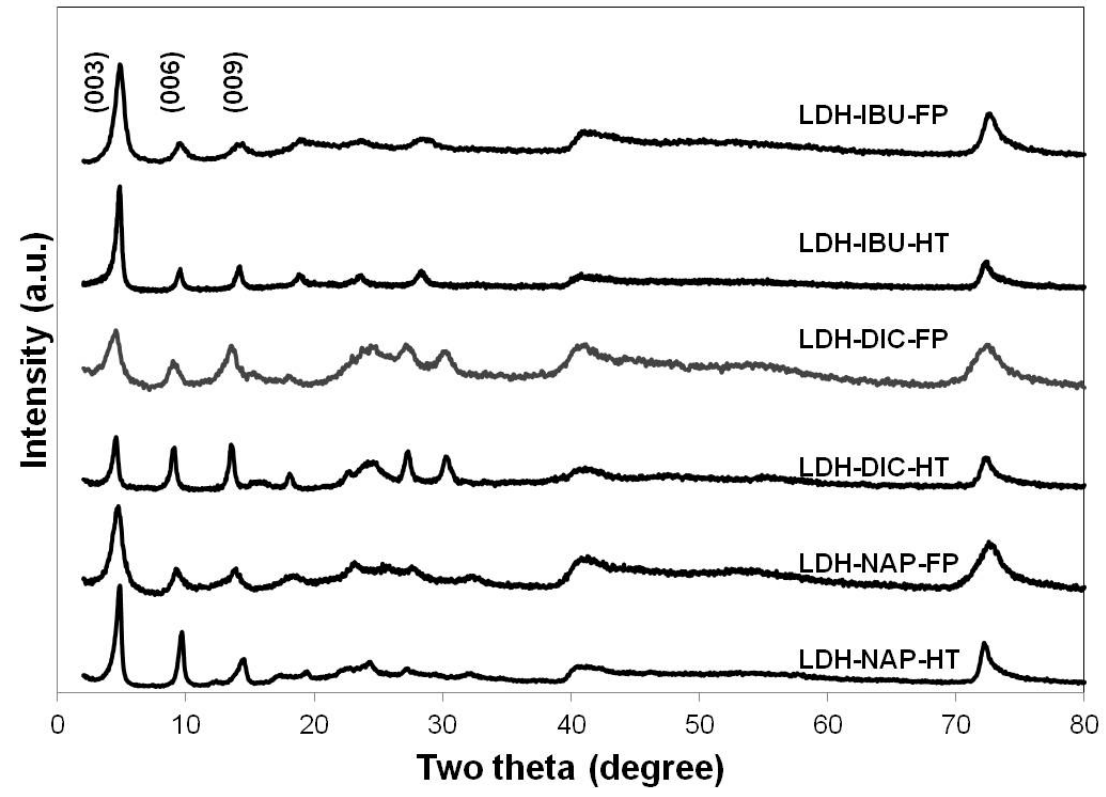

Table 2. Interlayer spacing and full width at half-maximum (FWHM) values.

\begin{tabular}{cccccc}
\hline Sample & FWHM (003) $\left(^{\circ}\right)$ & $\boldsymbol{d}_{\mathbf{0 0 3}}(\mathbf{n m})$ & $\boldsymbol{d}_{\mathbf{0 0 6}}(\mathbf{n m})$ & $\boldsymbol{d}_{\mathbf{0 0 9}}(\mathbf{n m})$ & $\boldsymbol{d}$ spacing $(\mathbf{n m})$ \\
\hline LDH-NAP-HT & 0.46 & 2.10 & 1.06 & 0.71 & 2.12 \\
LDH-NAP-FP & 0.88 & 2.16 & 1.09 & 0.74 & 2.19 \\
LDH-DIC-HT & 0.50 & 2.24 & 1.13 & 0.76 & 2.26 \\
LDH-DIC-FP & 0.80 & 2.23 & 1.12 & 0.76 & 2.25 \\
LDH-IBU-HT & 0.48 & 2.12 & 1.08 & 0.72 & 2.15 \\
LDH-IBU-FP & 0.76 & 2.10 & 1.09 & 0.71 & 2.14 \\
\hline
\end{tabular}

Calculated by averaging $d_{003}, 2 d_{006}$ and $3 d_{009}$, the interlayer spacings of the LDH-NAP-HT/FP, LDH-DIC-HT/FP and LDH-IBU-HT/FP were 2.12, 2.19, 2.26, 2.25, 2.15 and $2.14 \mathrm{~nm}$, respectively (Table 2), in correspondence with the interlayer spacing of LDH-NAP/DIC/IBU using various synthesis methods reported in the literature [14,25-30]. The same interlayer spacing of LDH-drug before and after hydrothermal treatment suggests that hydrothermal treatment did not affect the drug anion arrangement in the LDH interlayer. The enlarged interlayer spacing of LDH-drug compared with that of $\mathrm{LDH}-\mathrm{NO}_{3}(0.85 \mathrm{~nm})$ [26] indicated the intercalation of drug anions into the $\mathrm{LDH}$ interlayer. Assuming a thickness of $0.48 \mathrm{~nm}$ for the brucite-like layer of LDH [31], the gallery height of LDH-drug hybrids was $1.6-1.8 \mathrm{~nm}$. Based on DFT computational simulation and assuming that $\mathrm{COO}^{-}$was vertical to the brucite-like layer of $\mathrm{LDH}$, the length of the drug molecules, NAP, DIC and IBU, and their dihedral angle, $\theta$, in the LDH gallery were $1.2 \mathrm{~nm}\left(39^{\circ}\right), 1.5 \mathrm{~nm}\left(31^{\circ}\right)$ and $1.0 \mathrm{~nm}\left(41^{\circ}\right)$, respectively, as derived from the optimised geometries. Therefore, we believe that the drug molecule 
orientation in the LDH gallery was a bilayer model without overlapping, as shown in Figure 3. However, in Costantino and coworkers' work [29], the interlayer drug anion orientation was based on the principal axis of drug anions being perpendicular to the brucite-like layer, and shown as a partially interdigitated bilayer stacking model.

Figure 3. Schematic representation of the bilayer stacking of naproxen, diclofenac or ibuprofen anions between the LDH layers. The interlayer spacing, drug molecule length and dihedral angle, $\theta$, of each LDH-drug are indicated.
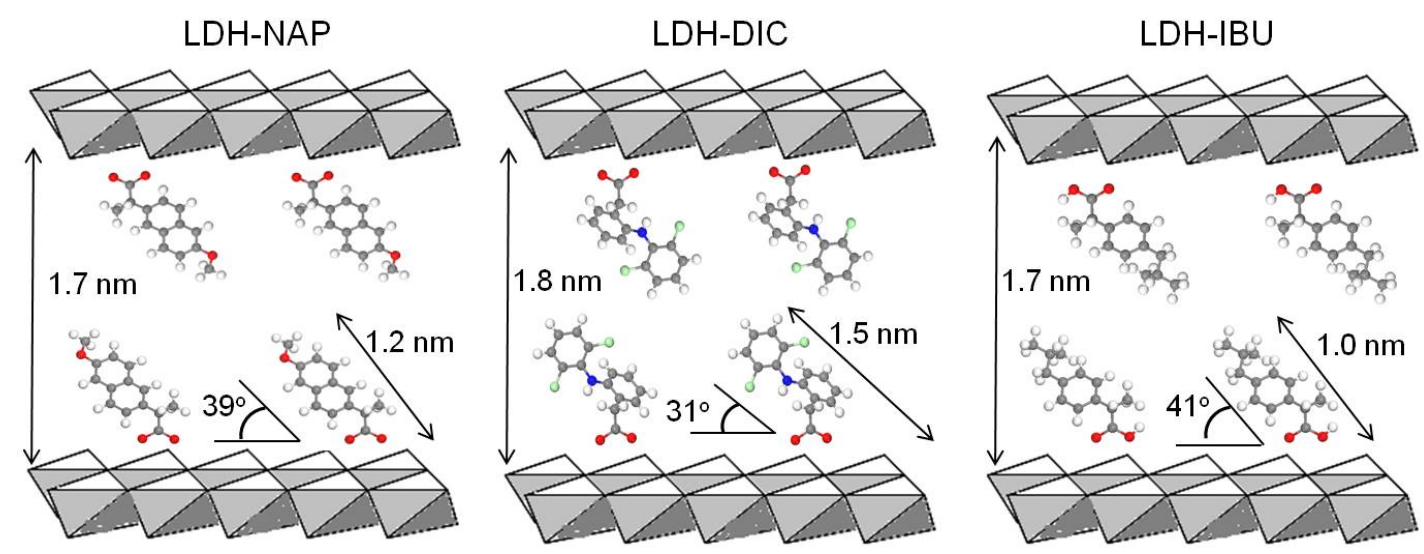

The intercalation of NAP, DIC or IBU drug molecules into LDHs was also confirmed by the FTIR spectra of LDH-drug samples (Figure 4). The spectra of LDH-drug samples showed the characteristic peaks of the LDH materials [9]: (1) the intense broad band around 3300-3500 $\mathrm{cm}^{-1}$ associated with the stretching vibration of $\mathrm{O}-\mathrm{H}$ in the brucite-like layer and water molecules; (2) the band at $544 \mathrm{~cm}^{-1}$ attributed to the $\mathrm{M}-\mathrm{O}$ and $\mathrm{M}-\mathrm{O}-\mathrm{H}$ stretching vibrations; and (3) the peak at $440 \mathrm{~cm}^{-1}$, particularly characteristic of $\mathrm{Mg}_{2} \mathrm{Al}-\mathrm{LDH}$ materials. Bands around 1570 and $1400 \mathrm{~cm}^{-1}$ in the LDH-drug spectra were attributed to the asymmetric and symmetric stretching vibrations of carboxylate, respectively $[27,32]$. The asymmetric carboxylate band moved to a lower frequency compared with the corresponding bands in drug-sodium salt, due to the strong electrostatic interactions between drug $\mathrm{COO}^{-}$groups and LDH layers. For example, $1580 \mathrm{~cm}^{-1}$ of NAP and $1570 \mathrm{~cm}^{-1}$ of DIC shifted to 1540 and $1550 \mathrm{~cm}^{-1}$, respectively, after intercalation to $\mathrm{LDH}$. There was no obvious difference between LDH-drug-HT and LDH-drug-FP in the FTIR spectrum, in accordance with the similar arrangement of drug anions in the LDH interlayer before and after hydrothermal treatment. In addition, other figure-printing IR peaks of drug molecules, attributed to the $\mathrm{C}-\mathrm{O}, \mathrm{C}=\mathrm{C}, \mathrm{C}-\mathrm{H}$ vibrations, remained unchanged after intercalation, which were not affected by the hydrothermal treatment either.

Table 3 lists the chemical composition of all LDH-drug samples. As expected, the molar ratio of $\mathrm{Mg}$ to $\mathrm{Al}$ in all samples was around 2.0 and slightly decreased after hydrothermal treatment, probably due to the slightly higher solubility of $\mathrm{Mg}$-hydroxide at the higher temperature. The drug loading capacity of LDH-NAP/DIC/IBU was $39.0 \%-47.4 \%$, close to or lower than that reported in the literature [14,25,27-30]. For example, Costantino and coworkers reported that the drug loading capacity of DIC and IBU in LDH was 55\% and 50\%, respectively [29]. The relatively higher drug loading capacity may result from: (1) the $\mathrm{NO}_{3}-\mathrm{LDH}$ having higher anion exchange capability than $\mathrm{Cl}$-LDH that we used in this work; and (2) carbonate contamination in our work. The chemical 
composition and drug loading capacity did not change after hydrothermal treatment, which was consistent with the observations on the crystal and chemical structure based on XRD and FTIR results.

Figure 4. Fourier transform infrared (FTIR) patterns for LDH-NAP-HT/FP, LDH-DIC-HT/FP and LDH-IBU-HT/FP.
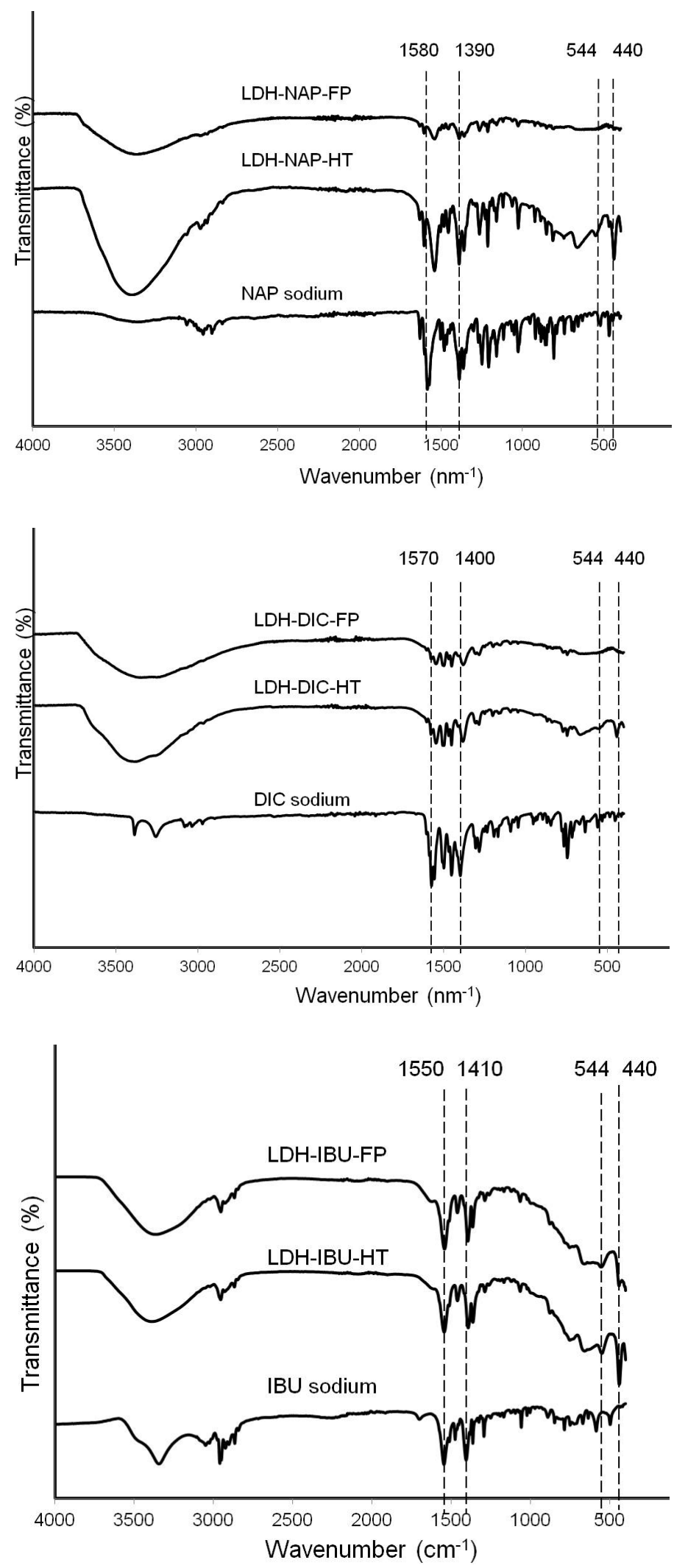
Table 3. Ratios of metal elements, drug loading capacity and predicted chemical structures of LDH-NAP-HT/FP, LDH-DIC-HT/FP and LDH-IBU-HT/FP.

\begin{tabular}{cccccccc}
\hline Sample & Mg (wt\%) & $\mathbf{A l}(\mathbf{w t} \%)$ & $\mathbf{N}(\mathbf{w t} \%)$ & $\mathbf{C}(\mathbf{w t} \%)$ & $\mathbf{H}(\mathbf{w t} \%)$ & $\begin{array}{c}\text { Mg/Al } \\
\text { molar ratio }\end{array}$ & $\begin{array}{c}\text { Drug loading } \\
\text { capacity (\%) }\end{array}$ \\
\hline LDH-NAP-HT & 10.2 & 6.26 & 0 & 36.5 & 5.09 & 1.81 & 42.1 \\
LDH-NAP-FP & 7.75 & 4.10 & 0 & 32.0 & 4.89 & 2.10 & 39.0 \\
LDH-DIC-HT & 11.0 & 5.75 & 2.44 & 32.1 & 4.04 & 2.12 & 45.9 \\
LDH-DIC-FP & 7.51 & 3.85 & 2.98 & 31.4 & 3.88 & 2.17 & 45.4 \\
LDH-IBU-HT & 11.1 & 6.98 & 0 & 33.0 & 6.10 & 1.76 & 47.4 \\
LDH-IBU-FP & 10.4 & 5.97 & 0 & 34.3 & 6.05 & 1.93 & 41.1 \\
\hline
\end{tabular}

\subsection{Drug Release Behaviours}

The drug release profiles of LDH-drug samples are shown in Figure 5. The drug anions were released from LDH-drug-HT/FP by exchange with phosphate anions in a sustained manner (Figure 5 and Figure S1 [33]). Within the first $8 \mathrm{~h}$, all LDH-drug samples had a burst release of drugs, which was assumed to quickly establish the therapeutic dose. Subsequently, the drug was continuously released at a much slower pace, and then, the release gradually approached the maximum amount in the next three days. The slower release rate allows the therapeutic dose to be retained for a longer period of time, thus potentially reducing the number of dosages applied on patients.

Figure 5. The release patterns of drugs from LDH-NAP-HT/FP, LDH-DIC-HT/FP and LDH-IBU-HT/FP in pH 7.4 PBS medium.
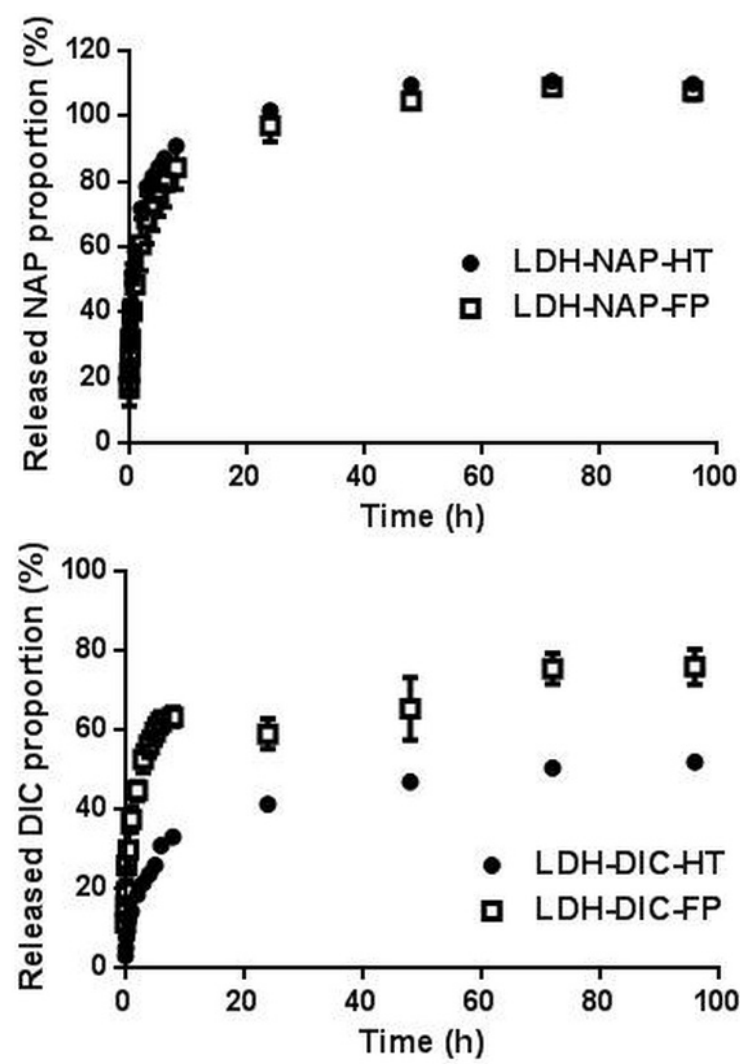
Figure 5. Cont.

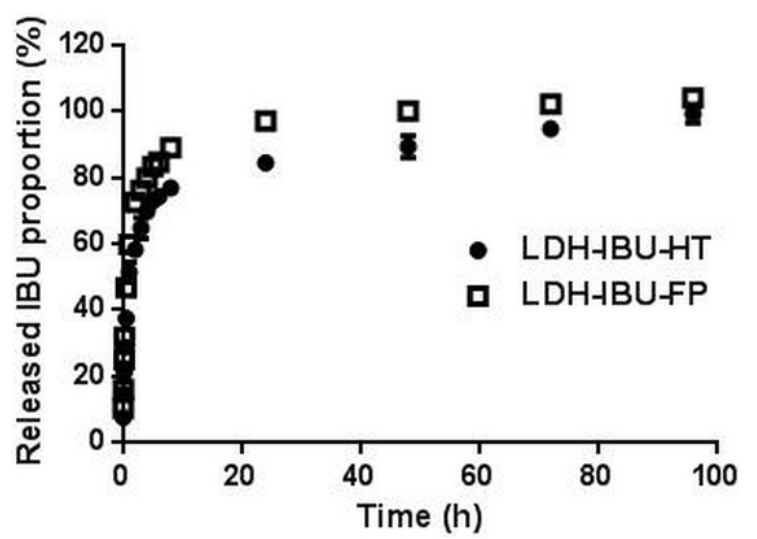

In general, LDH-NAP/DIC/IBU released the drug at a slower rate than that reported previously $[25,28,34]$. The time required to release $50 \%$ of drugs $\left(T_{50}\right)$ from LDH-NAP/DIC/IBU (either being hydrothermally treated or not) was greater than $0.5 \mathrm{~h}$, as shown in Table 4 . In comparison, a much faster release rate $\left(T_{50}=1 \mathrm{~min}\right)$ from $\mathrm{LiAl}_{2}-\mathrm{LDH}-\mathrm{NAP} / \mathrm{DIC} / \mathrm{IBU}$ in $\mathrm{pH} 4$ or 7 PBS reported by O'Hare and coworkers may be caused by the LDH matrix partly dissolving under pH 4 or 7 [34]. Gunawan and Xu demonstrated that 50\% of IBU was released from MgAl-LDH for about $1 \mathrm{~h}$, comparable to that of LDH-IBU-HT $\left(T_{50}=1 \mathrm{~h}\right)$. However, $90 \%$ of the drug release reported by Gunawan and $\mathrm{Xu}$ required about $2.5 \mathrm{~h}$, which was much faster than what we observed from LDH-IBU-HT $\left(T_{90}=48 \mathrm{~h}\right.$; Table 4$)$. This may also result from the release medium with a lower $\mathrm{pH}$ (pH 7 PBS) in Gunawan and Xu's study [25]. Moreover, the drug release of LDH-DIC-HT in this work was much slower than that reported by Perioli et al. In their study, $80 \%$ of the loaded DIC was released at the first hour [28]. Considering the similar particle size (around $200 \mathrm{~nm}$ and the aggregated ones around $300 \mathrm{~nm}$ ) and release medium $\mathrm{pH}$ value (7.4-7.5), the difference in the drug release between these two cases is not clear, so far.

Table 4. Key parameters in LDH-drug release. $T_{50}$ and $T_{90}$ are the times required to release $50 \%$ and $90 \%$ of drugs from LDH-drug. The lipophilicity $(\log P)$ and molecular weight of drugs are also listed [32,34].

\begin{tabular}{ccccc}
\hline Sample & $\boldsymbol{T}_{\mathbf{5 0}}(\mathbf{h})$ & $\boldsymbol{T}_{\mathbf{9 0}}(\mathbf{h})$ & $\log \boldsymbol{P}$ of drug & Molecular weight of drug \\
\hline LDH-NAP-HT & 2.5 & 8 & \multirow{2}{*}{230} \\
LDH-NAP-FP & 6 & 15 & 3.2 & \multirow{2}{*}{295} \\
\hline LDH-DIC-HT & $>96$ & $>96$ & 4.75 & \multirow{2}{*}{206} \\
LDH-DIC-FP & 56 & $>96$ & & \\
\hline LDH-IBU-HT & 1 & 48 & 3.5 & \\
LDH-IBU-FP & 0.5 & 1 & &
\end{tabular}

The drug release from LDH-drug-HT was slower than that from corresponding LDH-drug-FP at every time point (Figure 5), in correspondence with Gunawan and Xu's findings [25]. More specifically, the release profiles of LDH-IBU-HT/FP and LDH-DIC-HT/FP were clearly separated by approximately $10 \%$ and $20 \%$ after the burst release, while the release profiles of LDH-NAP-HT/FP were almost overlapped at most points. The slower release rate from LDH-IBU/DIC-HT composites in the former 
case could be attributed to the larger crystal size (Table 1 and Figure 1), which made a longer diffusion path of the drug from the LDH matrix to the release medium.

In comparison with the release percentage of three drugs from the LDH matrix, LDH-DIC had a slower DIC release than LDH-NAP and LDH-IBU. For example, LDH-DIC-FP released approximately $62 \%$ and $76 \%$ of the loaded drug after 8 and 96 h, respectively, compared with LDH-NAP-FP (84\% and $\sim 100 \%$ ) and LDH-IBU-FP (77\% and $100 \%$ ) (Table 4 and Figure 5). The difference in the release rate of different drugs was mainly determined by the interactions of the anion with the LDH hydroxide layer and the hydrophobic interactions among drug molecules in the interlayer. Since each drug anion had one carboxylate group and the $\mathrm{MgAl}$ ratio was close to 2.0, the interactions between the drug anion and the LDH hydroxide layer should be similar. However, the hydrophobic interactions among drug molecules would be very much different. As shown in Table 4, the lipophilicity of three drugs, e.g., $\log P$ (NAP: 3.2; DIC: 4.75 and IBU 3.5), represented the inter-molecular hydrophobic interaction (Table 4) $[35,36]$. DIC had a larger $\log P$ and a stronger inter-molecular hydrophobic interaction, and thus, its release had to overcome a higher barrier, leading to a slower release rate and a smaller release percentage. As reported by Xu and Braterman [37], the inter-molecular hydrophobic interaction enabled the affinity of dodecylbenzene sulfonate for $\mathrm{LDH}$ comparable with that of $\mathrm{CO}_{3}{ }^{2-}$ for $\mathrm{LDH}$. In addition, the heavier molecular weight of DIC (Table 4) would cause DIC to diffuse more slowly.

\section{Conclusions}

This paper demonstrated that the particle size of the drug delivery carrier and the physicochemical properties of drugs determine the drug release from drug-intercalated LDHs. Hydrothermal treatment increased the LDH particle size and crystallinity, and the resultant larger size led to a decreased drug release rate. Thus, hydrothermal treatment can be considered an approach to control the LDH-drug particle size and the drug release rate, in addition to the drug molecular physicochemical properties.

\section{Acknowledgments}

The authors acknowledge Chenghua Sun from Monash University, Australia, for the drug orientation model and relevant calculation, the financial support from the National Health and Medical Research Council of Australia (NHMRC) through the NHMRC Early Career Fellowship (APP1073591) and the Australian Research Council (ARC) through the Discovery Project (DP120104792) and the Future Fellowship (FT120100813).

\section{Author Contributions}

The reported research was designed and written by Dr Zi Gu and Associate Professor Zhi Ping Xu, and conducted by Miss Aihua $\mathrm{Wu}, \mathrm{Dr} \mathrm{Zi} \mathrm{Gu}$, and Dr Li Li. All the authors in the author list were involved in data discussion and analysis.

\section{Conflicts of Interest}

The authors declare no conflict of interest. 


\section{References}

1. Braterman, P.S.; Xu, Z.P.; Yarberry, F. Handbook of Layered Materials; Auerbach, S.M., Carrado, K.A., Dutta, P.K., Eds.; Marcel Dekker: New York, NY, USA, 2004; pp. 373-474.

2. Xu, Z.P.; Lu, G.Q. Layered double hydroxide nanomaterials as potential cellular drug delivery agents. Pure Appl. Chem. 2006, 78, 1771-1779.

3. Xu, Z.P.; Zeng, Q.H.; Lu, G.Q.; Yu, A.B. Inorganic nanoparticles as carriers for efficient cellular delivery. Chem. Eng. Sci. 2006, 61, 1027-1040.

4. Xu, Z.P.; Niebert, M.; Porazik, K.; Walker, T.L.; Cooper, H.M.; Middelberg, A.P.J.; Gray, P.P.; Bartlett, P.F.; Lu, G.Q. Subcellular compartment targeting of layered double hydroxide nanoparticles. J. Control. Release 2008, 130, 86-94.

5. Huang, H.C.; Barua, S.; Sharma, G.; Dey, S.K.; Rege, K. Inorganic nanoparticles for cancer imaging and therapy. J. Control. Release 2011, 155, 344-357.

6. Li, A.; Qin, L.L.; Wang, W.R.; Zhu, R.R.; Yu, Y.C.; Liu, H.; Wang, S.L. The use of layered double hydroxides as DNA vaccine delivery vector for enhancement of anti-melanoma immune response. Biomaterials 2011, 32, 469-477.

7. Oh, J.M.; Park, M.; Kim, S.T.; Jung, J.Y.; Kang, Y.G.; Choy, J.H. Efficient delivery of anticancer drug MTX through MTX-LDH nanohybrid system. J. Phys. Chem. Solids 2006, 67, 1024-1027.

8. Gu, Z.; Rolfe, B.E.; Xu, Z.P.; Thomas, A.C.; Campbell, J.H.; Lu, G.Q. Enhanced effects of low molecular weight heparin intercalated with layered double hydroxide nanoparticles on rat vascular smooth muscle cells. Biomaterials 2010, 31, 5455-5462.

9. Gu, Z.; Thomas, A.C.; Xu, Z.P.; Campbell, J.H.; Lu, G.Q. In vitro sustained release of LMWH from MgAl-layered double hydroxide nanohybrids. Chem. Mater. 2008, 20, 3715-3722.

10. Gu, Z.; Rolfe, B.E.; Thomas, A.C.; Campbell, J.H.; Lu, G.Q.; Xu, Z.P. Cellular trafficking of low molecular weight heparin incorporated in layered double hydroxide nanoparticles in rat vascular smooth muscle cells. Biomaterials 2011, 32, 7234-7240.

11. Gu, Z.; Rolfe, B.E.; Xu, Z.P.; Campbell, J.H.; Lu, G.Q.; Thomas, A.C. Antibody-targeted drug delivery to injured arteries using layered double hydroxide nanoparticles. Adv. Healthc. Mater. 2012, 1, 669-673.

12. Bonina, F.P.; Giannossi, M.L.; Medici, L.; Puglia, C.; Summa, V.; Tateo, F. Diclofenac-hydrotalcite: In vitro and in vivo release experiments. Appl. Clay Sci. 2008, 41, 165-171.

13. Carriazo, D.; del Arco, M.; Martin, C.; Ramos, C.; Rives, V. Influence of the inorganic matrix nature on the sustained release of naproxen. Microporous Mesoporous Mater. 2010, 130, 229-238.

14. Del Arco, M.; Gutierrez, S.; Martin, C.; Rives, V.; Rocha, J. Synthesis and characterization of layered double hydroxides (LDH) intercalated with non-steroidal anti-inflammatory drugs (NSAID). J. Solid State Chem. 2004, 177, 3954-3962.

15. Li, B.X.; He, J.; Evans, D.G.; Duan, X. Inorganic layered double hydroxides as a drug delivery system-intercalation and in vitro release of fenbufen. Appl. Clay Sci. 2004, 27, 199-207.

16. Xu, Z.P.; Stevenson, G.; Lu, C.Q.; Lu, G.Q. Dispersion and size control of layered double hydroxide nanoparticles in aqueous solutions. J. Phys. Chem. B 2006, 110, 16923-16929.

17. Labajos, F.M.; Rives, V.; Ulibarri, M.A. Effect of hydrothermal and thermal treatments on the physicochemical properties of Mg-Al hydrotalcite-like materials. J. Mater. Sci. 1992, 27, 1546-1552. 
18. Kohn, W.; Sham, L.J. Self-consistent equations including exchange and correlation effects. Phys. Rev. 1965, 140, 1133-1138.

19. Perdew, J.P.; Burke, K.; Ernzerhof, M. Generalized gradient approximation made simple. Phys. Rev. Lett. 1996, 77, 3865-3868.

20. Delley, B. An all-electron numerical method for solving the local density functional for polyatomic molecules. J. Chem. Phys. 1990, 92, 508-517.

21. Delley, B. From molecules to solids with the DMol(3) approach. J. Chem. Phys. 2000, 113, 7756-7764.

22. Xu, Z.P.; Stevenson, G.S.; Lu, C.Q.; Lu, G.Q.; Bartlett, P.F.; Gray, P.P. Stable suspension of layered double hydroxide nanoparticles in aqueous solution. J. Am. Chem. Soc. 2006, 128, 36-37.

23. Musumeci, A.W.; Xu, Z.P.; Smith, S.V.; Minchin, R.F.; Martin, D.J. Layered double hydroxide nanoparticles incorporating terbium: Applicability as a fluorescent probe and morphology modifier. J. Nanopart. Res. 2010, 12, 111-120.

24. Xu, Z.P.; Braterman, P.S. Synthesis, structure and morphology of organic layered double hydroxide (LDH) hybrids: Comparison between aliphatic anions and their oxygenated analogs. Appl. Clay Sci. 2010, 48, 235-242.

25. Gunawan, P.; Xu, R. Direct control of drug release behavior from layered double hydroxides through particle interactions. J. Pharm. Sci. 2008, 97, 4367-4378.

26. Wei, M.; Shi, S.X.; Wang, J.; Li, Y.; Duan, X. Studies on the intercalation of naproxen into layered double hydroxide and its thermal decomposition by in situ FT-IR and in situ HT-XRD. J. Solid State Chem. 2004, 177, 2534-2541.

27. Ambrogi, V.; Fardella, G.; Grandolini, G.; Perioli, L.; Tiralti, M.C. Intercalation compounds of hydrotalcite-like anionic clays with anti-inflammatory agents, II: Uptake of diclofenac for a controlled release formulation. AAPS PharmSciTech 2002, 3, 77-82.

28. Perioli, L.; Posati, T.; Nocchetti, M.; Bellezza, F.; Costantino, U.; Cipiciani, A. Intercalation and release of antiinflammatory drug diclofenac into nanosized ZnAl hydrotalcite-like compound. Appl. Clay Sci. 2011, 53, 374-378.

29. Costantino, U.; Ambrogi, V.; Nocchetti, M.; Perioli, L. Hydrotalcite-like compounds: Versatile layered hosts of molecular anions with biological activity. Microporous Mesoporous Mater. 2008, 107, 149-160.

30. Ambrogi, V.; Fardella, G.; Grandolini, G.; Perioli, L. Intercalation compounds of hydrotalcite-like anionic clays with antiinflammatory agents-I. Intercalation and in vitro release of ibuprofen. Int. J. Pharm. 2001, 220, 23-32.

31. Drezdzon, M.A. Synthesis of isopolymetalate-pillared hydrotalcite via organic-anion-pillared precursors. Inorg. Chem. 1988, 27, 4628-4632.

32. Rezvani, Z.; Sarkarat, M. Synthesis and characterization of magnetic composites: Intercalation of naproxen into $\mathrm{Mg}-\mathrm{Al}$ layered double hydroxides coated on $\mathrm{Fe}_{3} \mathrm{O}_{4}$. Z. Anorg. Allg. Chem. 2012, $638,874-880$.

33. Khan, A.I.; Lei, L.X.; Norquist, A.J.; O'Hare, D. Intercalation and controlled release of pharmaceutically active compounds from a layered double hydroxide. Chem. Commun. 2001, doi:10.1039/B106465G. 
34. Fini, A.; Fazio, G.; Feroci, G. Solubility and solubilization properties of non-steroidal anti-inflammatory drugs. Int. J. Pharm. 1995, 126, 95-102.

35. Paavola, A.; Yliruusi, J.; Rosenberg, P. Controlled release and dura mater permeability of lidocaine and ibuprofen from injectable poloxamer-based gels. J. Control. Release 1998, 52, 169-178.

36. Xu, Z.P.; Braterman, P.S. High affinity of dodecylbenzene sulfonate for layered double hydroxide and resulting morphological changes. J. Mater. Chem. 2003, 13, 268-273.

37. Shimamura, A.; Jones, M.I.; Kanezaki, E.; Metson, J.B. Complete desporption of interlayer hydrogen phosphate in $\mathrm{Mg} / \mathrm{Al}$-layered double hydroxides by means of anion exchange with 1-octanesulfonate. J. Mater. Sci. 2012, 47, 1142-1147.

(C) 2014 by the authors; licensee MDPI, Basel, Switzerland. This article is an open access article distributed under the terms and conditions of the Creative Commons Attribution license (http://creativecommons.org/licenses/by/3.0/). 